\title{
Evaluation of tissue culture raised crops of sugarcane as initial source of seed cane at matahara sugar estate, Ethiopia
}

\begin{abstract}
Tissue culture technology, if properly incorporated into the system of sugarcane production, has better rate of propagation, more cane and sugar yield than the conventional seed source and hence makes the sugar industry more profitable. With this view, the Ethiopian Sugar Industry uses this technology since 2012. However, there was no data on the magnitude of the advantage of this technology under the Ethiopian Sugar Estates. Thus, an experiment was carried out at Matahara Sugar Estate to compare the two seed sources. Comparison of the two seed sources at plant cane stage showed that tissue culture seed sources showed less performance in average single cane weight $(\mathrm{kg})$, cane yield (ton/ha) and sugar yield (ton/ha). However, there is no significant difference in stalk height $(\mathrm{cm})$, stalk diameter $(\mathrm{cm})$, stalk population (number/ha), sucrose percent cane (ton/ha), Pol (\%), Brix and purity (\%) between the two sources for the three sugarcane genotypes tested. From this result, it can be deduced that regardless of comparison of their costs, sugarcane plants from conventional seed sources performanced better than tissue culture derived plants. The reduced performance of tissue culture seed source could probably be due to the then non standard secondary acclimatization stage where the plantlets face additional stress on top of primary acclimation. In addition, tissue culture plantlets may require separate agronomic management practice for better yield at initial seed cane stage. Furthermore, lack of quality control of tissue culture plants for their genetic fidelity and disease freeness at different micro propagation stages may have its own performance limitations.
\end{abstract}

Keywords: seed source, tissue culture, conventional, sugarcane genotypes, NCo334, B52-298
Volume 3 Issue 2 - 2017

\author{
Belay Tolera, Dereje Shimelis \\ Wonji Research \& Development Center, Ethiopia \\ Correspondence: Belay Tolera, Ethiopian Sugar Corporation \\ Research and Development Center,Variety Development \\ Research Directorate, Biotechnology Research Team, Wonji \\ Research \& Development Center: P.O.Box: I5, Ethiopia, Tel \\ +25I-910-181644, Fax +25I-222-200-I44, \\ Email belaytolera@yahoo.com
}

Received: October 18, 2016 | Published: May 15, 2017

\section{Introduction}

Sugarcane (Saccharum officinarum L.) is a monocotyledonous crop plant that belongs to the grass family, poaceae. ${ }^{1}$ It is an octaploid crop with chromosome number of $2 \mathrm{n}=800^{2-3}$ It is a tall growing perennial grass that tillers at the base, grows three to four meters tall with about five $\mathrm{cm}$ in diameter. ${ }^{4}$ Today, the crop is grown in over 120 countries and $50 \%$ of the production occurs in Brazil and India where the annual global sugar production was estimated to be more than 174.3 million tones, accounting nearly for $70 \%$ of the production. ${ }^{5,6}$ Sugarcane has been cultivated in the tropical and subtropical regions of the world for its multiple uses. The sugar juice is used for making sugar. Molasses (thick syrupy residue) is used in the production of ethanol (blended for motor fuel) and as livestock feed. The bagasse (fibrous portion) is burned to provide heat and electricity for sugar mills (excess heat co-generated used for electricity and diverted to national grid). Fine sediments from cane juice extraction (press mud) mixed with ash from burned bagasse form filter cake which is used as organic fertilizer and can tops used as fuel wood and animal feed. Sugarcane is an important commercial cash and industrial crop in Ethiopia. The sugar industry in Ethiopia has great contribution to the socio-economy of the country in many ways. The contributions are concerned with production and consumption of sugar, income generation, employment creation, revenue contribution, electric power contribution, skill and knowhow development, capital formation, agriculture and other industries development, urbanization and market development benefit, provision of access to health, education and road facilities. Hence, improving sugarcane production capacity has paramount importance in enhancing the economic prosperity of the nation. Sugarcane plants raised through seeds (fuzz) are highly heterozygous and used only for breeding purpose. As a result, for commercial propagation, it is propagated vegetatively through stem cuttings (conventional propagation). However, the conventional propagation method where stem cuttings with two to three buds used as planting material has various limitations. The multiplication rate of sugarcane planting material is very slow (1:7-1:10). Using this method, availability of adequate quantity quality planting material of the available commercial cane varieties and commercialization of improved introduced and adapted sugarcane varieties within a short time is the major limiting factor to the Ethiopian Sugar Industry for expansion of existing sugarcane farms and planting of new sugar development projects. Moreover, the yield of the existing few commercial cane varieties is declining sharply and some productive varieties were obsolete due to lack of alternative technologies for disease cleansing and rejuvenation of old sugarcane varieties used for about 50 years. Plant tissue culture (micropropagation) is a tool for obtaining rapid rejuvenation and mass multiplication of disease free, true to type and uniform planting materials of sugarcane. Moreover, tissue culture raised sugarcane plants were reported to give superior cane and sugar yield. Accordingly, micro propagated sugarcane plantlets are superior in cane height, stalk girth, number of millable canes per clump, cane yield per hectare with broader and green leaves as compared to their donors. ${ }^{7}$ The same authors also reported that tissue culture raised sugarcane plantlets have more percent sugar recovery as compared 
to their donor plants propagated through conventional method. Tissue culture raised sugarcane crops showed $32 \%$ and $8 \%$ more cane yield in plant cane and first ratoon respectively; and $33 \%$ and $12 \%$ more sugar yield in the plant crop and first ratoon respectively Similarly, in vitro raised sugarcane plants showed $13.2 \%$ increase in cane yield and $11.03 \%$ more sugar recovery as compared to conventionally propagated similar varieties of sugarcane under similar agronomic management system. ${ }^{8}$ However, there is limited information on field performance of in vitro micropropagated sugarcane plantlets planted under Ethiopian Sugar Estates. Moreover, the yield advantage and profitability of using this technology need to be clearly known. Thus, it is imperative to compare the field performance of the two seed sources. Therefore, the objective of this study was to evaluate the field performance of tissue culture raised sugarcane plants as compared to the conventional raised sugarcane plants of the same genotype under similar agronomic management practices.

\section{Materials and methods}

The study was carried out at Metahara sugarcane plantation, located at Eastern part of the country, at about $200 \mathrm{kms}$ away from Addis Ababa, Ethiopia. Metahara Sugar estate is situated at $80^{\circ} 53^{\prime} \mathrm{N}$ latitude and $390^{\circ} 52^{\prime} \mathrm{E}$ longitudes at an altitude of 950 mas. The area has a semi arid climatic condition. The experimental materials were in vitro micro propagated and conventionally raised sugarcane varieties of NCO-334, B52-298 and N14. The primary acclimatized plantlets of these sugarcane varieties were delivered from Mekelle Technology Institute Tissue Culture Laboratory and secondary acclimatized at Metahara sugar estate and planted at the experimental fields. The experimental design was Randomized Complete Block design and the plot size was six furrows with 5 meter length $\left(43.5 \mathrm{~m}^{2}\right)$. Three sugarcane genotypes raised from tissue culture and conventional propagation method were planted on two major soil types forming $3 \times 2 \times 2=12$ treatment combinations. Each treatment was replicated three times. Data on stalk height, single cane weight $(\mathrm{kg})$, diameter $(\mathrm{cm})$, stalk population (Number/plot), cane yield (ton/ha), sucrose percent cane, sugar yield (ton/ha), Pol(\%), Brix and purity (\%) were collected. The collected data were subjected to analysis of variance (ANOVA) using SAS statistical software (version 9.2) system and separation of significant means were done using REGWQ multiple range test.

\section{Result and discussion}

Analysis of variance showed that the interaction effects of the three factors; genotype, seed source and soil type (Genotype* Seed source* Soil type $=\mathrm{P} \leq 0.05$ ) were highly significant for the response variables stalk diameter $(\mathrm{cm})$, estimated cane yield (ton/ha), sucrose percent cane $(\%), \operatorname{Pol}(\%)$ and Brix while stalk height $(\mathrm{cm})$, stalk population (Number/ha), estimated sugar yield (ton/ha) and purity (\%) were dependent on the interaction effects of genotype and seed source (Genotype* Seed source $=\mathrm{P} \leq 0.05$ ) . In addition, the interaction of genotype by soil type has a highly significant effect on purity (\%). However, result from ANOVA showed that average single cane weight $(\mathrm{kg})$ is dependent only on seed source (Table 1). Regardless of the soil type and genotypes, comparison of the two seed sources (tissue culture Vs conventional) indicated that conventional seed sources at plant cane stage had better average single cane weight (kg), cane yield (ton/ ha) and sugar yield (ton/ha) while there is no significant difference between the two seed sources in stalk height $(\mathrm{cm})$, stalk Diameter $(\mathrm{cm})$, stalk population (Number/ha), sucrose percent cane (\%), Pol $(\%)$, Brix and purity (\%) (Table 2). The current result is in contrast with the findings of ${ }^{9}$ who reported increased stalk population and reduced cane and sugar yield in tissue culture seed over conventional seed sources and the findings of ${ }^{7,8,10}$ who reported better field performance of tissue culture over conventional seed sources. Similarly, the current result is in contrast with our findings at Tendaho Sugar Development Project where tissue culture seed sources produced four times more cane yield(ton/ha) as compared to their donors from conventional seed sources. ${ }^{11}$ Regardless of the soil type and the seed sources, comparison of the sugarcane genotypes show that sugarcane genotype N14 gave the highest stock population (Number/ha), cane yield (ton/ ha), sugar yield (ton/ha) and purity (\%) than genotypes $\mathrm{NCo}-334$ and B52-298. Similarly, the genotype B52-298 showed the highest stalk diameter $(\mathrm{m})$, sucrose percent cane $(\%)$ and Pol $(\%)$ and Brix than the other genotypes while $\mathrm{NCo}-334$ has best in stalk height $(\mathrm{m})$ than the other two tested genotypes (Table 3). However; there is no significant difference between the three genotypes for the response variable single cane weight $(\mathrm{kg})$ at seed cane harvest age of 10 months. In sugarcane genotype B52-298, comparison of the two seed sources on Luvisol revealed that tissue culture raised plants gave better Sucrose percent cane (\%) (13.23 \pm 0.63$)$; Pol (\%) (18.62 \pm 0.76$)$ and Brix (20.23 \pm 0.60$)$ than the conventional seed source while the conventional seed source gave better cane yield (ton/ha) (221.279 $\pm 2.83 \mathrm{~d})$ than tissue culture seed source. However; there is no significant difference in stalk diameter $(\mathrm{cm})$ of the two seed sources of the genotype on Luvisol. Again, for the same genotype (B52-298) on Vertisol, the conventional

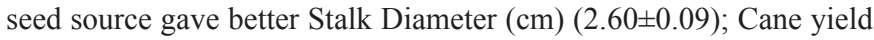
(ton/ha) (220.114 \pm 2.83 ); sucrose percent cane (11.62 \pm 0.63$)$; Pol\% $(16.81 \pm 0.76)$ and Brix $(18.97 \pm 0.60)$ than tissue culture seed source (Table 4).

Table I ANOVA for comparison of micro propagated and conventional seed sources of three sugarcane genotypes

\begin{tabular}{|c|c|c|c|c|c|c|c|c|c|c|c|}
\hline \multirow[b]{2}{*}{$\begin{array}{l}\text { Source of } \\
\text { variation }\end{array}$} & \multirow[b]{2}{*}{ DF } & \multicolumn{10}{|c|}{ Mean squares } \\
\hline & & $\begin{array}{l}\text { Height } \\
\text { (m) }\end{array}$ & $\begin{array}{l}\text { Single } \\
\text { cane } \\
\text { weight } \\
(\mathbf{k g})\end{array}$ & $\begin{array}{l}\text { Diameter } \\
(\mathrm{cm})\end{array}$ & $\begin{array}{l}\text { Stock } \\
\text { population } \\
\text { (Numberl } \\
\text { ha) }\end{array}$ & $\begin{array}{l}\text { Cane } \\
\text { yield } \\
\text { (ton/ha) }\end{array}$ & $\begin{array}{l}\text { Sucrose } \\
\text { percent } \\
\text { cane } \\
(\%)\end{array}$ & $\begin{array}{l}\text { Sugar } \\
\text { yield } \\
\text { (ton/ } \\
\text { ha) }\end{array}$ & $\begin{array}{l}\text { Pol } \\
(\%)\end{array}$ & Brix & Purity \\
\hline Genotype & 2 & $0.369 * *$ & $0.022 \mathrm{~ns}$ & $0.161 * *$ & $74524 I^{* * *}$ & $|6395.3|^{* *}$ & $4.818^{* *}$ & $8914^{* *}$ & $8.812^{* *}$ & $10.2264^{* * *}$ & $8.30 \mathrm{~ns}$ \\
\hline $\begin{array}{l}\text { Seed } \\
\text { Source }\end{array}$ & I & $0.250 \mathrm{~ns}$ & $0.467 * * *$ & $0.017 \mathrm{~ns}$ & $270344.8 n s$ & $126250 * *$ & $0.00 \mathrm{Ins}$ & $15074 * * *$ & $0.004 \mathrm{~ns}$ & $0.0004 n s$ & $0.04 n s$ \\
\hline $\begin{array}{l}\text { Genotype* } \\
\text { Seed } \\
\text { Source }\end{array}$ & 2 & $0.352^{* *}$ & $0.064 \mathrm{~ns}$ & $0.104 * *$ & $781172^{* *}$ & $|07507| * *$ & $0.996 \mathrm{~ns}$ & $5486 * *$ & $0.938 \mathrm{~ns}$ & $0.2794 \mathrm{~ns}$ & $15.82 * *$ \\
\hline
\end{tabular}


Table Continued..

\begin{tabular}{|c|c|c|c|c|c|c|c|c|c|c|c|}
\hline \multirow[b]{2}{*}{$\begin{array}{l}\text { Source of } \\
\text { variation }\end{array}$} & \multirow[b]{2}{*}{ DF } & \multicolumn{10}{|c|}{ Mean squares } \\
\hline & & $\begin{array}{l}\text { Height } \\
\text { (m) }\end{array}$ & $\begin{array}{l}\text { Single } \\
\text { cane } \\
\text { weight } \\
\text { (kg) }\end{array}$ & $\begin{array}{l}\text { Diameter } \\
(\mathrm{cm})\end{array}$ & $\begin{array}{l}\text { Stock } \\
\text { population } \\
\text { (Numberl } \\
\text { ha) }\end{array}$ & $\begin{array}{l}\text { Cane } \\
\text { yield } \\
\text { (ton/ha) }\end{array}$ & $\begin{array}{l}\text { Sucrose } \\
\text { percent } \\
\text { cane } \\
(\%)\end{array}$ & $\begin{array}{l}\text { Sugar } \\
\text { yield } \\
\text { (ton/ } \\
\text { ha) }\end{array}$ & $\begin{array}{l}\text { Pol } \\
\text { (\%) }\end{array}$ & Brix & Purity \\
\hline Soil type & I & $\begin{array}{l}0.066 \\
\mathrm{~ns}\end{array}$ & $0.026 \mathrm{~ns}$ & $0.025 \mathrm{~ns}$ & $10830 \mid * *$ & $2828.83 * *$ & $1.818 \mathrm{~ns}$ & $8440 * *$ & $0.608 \mathrm{~ns}$ & $0.6834 \mathrm{~ns}$ & $82.45 * * *$ \\
\hline $\begin{array}{l}\text { Genotype* } \\
\text { Soil type }\end{array}$ & 2 & $\begin{array}{l}0.010 \\
\mathrm{~ns}\end{array}$ & $0.002 \mathrm{~ns}$ & $0.015 \mathrm{~ns}$ & $655172 \mathrm{~ns}$ & $1310.34 \mathrm{~ns}$ & $2.132 \mathrm{~ns}$ & $36 \mathrm{Ins}$ & $3.755 \mathrm{~ns}$ & $3.6100 * *$ & $2.26 * *$ \\
\hline $\begin{array}{l}\text { Seed } \\
\text { Source* } \\
\text { Soil type }\end{array}$ & I & $\begin{array}{l}0.020 \\
\text { ns }\end{array}$ & $0.006 \mathrm{~ns}$ & $0.011 \mathrm{~ns}$ & $23793 \mathrm{~ns}$ & I42.76ns & $5.437 * *$ & $2850 \mathrm{~ns}$ & $7.896 * *$ & $4.5227^{* *}$ & I6.78ns \\
\hline $\begin{array}{l}\text { Genotype* } \\
\text { Seed } \\
\text { Source* } \\
\text { Soil type }\end{array}$ & 2 & $\begin{array}{l}0.016 \\
\mathrm{~ns}\end{array}$ & $0.094 \mathrm{~ns}$ & $0.099 * *$ & I74000ns & $16356 * *$ & $4.884 * *$ & $2044 n s$ & $7.384 * *$ & $5.8866 * *$ & $11.35 \mathrm{~ns}$ \\
\hline CV (\%) & & II.8I & 13.1 & 6.22 & 14.4 & 18.8 & 9.92 & 18.8 & 8.31 & 5.89 & 2.48 \\
\hline
\end{tabular}

$* *: \mathrm{P}>0.00$ I but $<0.05, * * *: \mathrm{P}<0.00 \mathrm{I}$ ns: $\mathrm{p}>0.05$ at $5 \%$ probability level.

Table 2 Response of sugarcane genotypes to the two seed sources

\begin{tabular}{|c|c|c|c|c|c|c|c|c|c|c|}
\hline \multirow[b]{2}{*}{$\begin{array}{l}\text { Seed } \\
\text { source }\end{array}$} & \multicolumn{10}{|c|}{ Tukey's standardized range(HSD) grouping } \\
\hline & $\begin{array}{l}\text { Stalk } \\
\text { Height } \\
\text { (m) }\end{array}$ & $\begin{array}{l}\text { Single } \\
\text { cane } \\
\text { weight } \\
(\mathrm{kg})\end{array}$ & $\begin{array}{l}\text { Diameter } \\
c(m)\end{array}$ & $\begin{array}{l}\text { Stock } \\
\text { population } \\
\text { (Number/ha) }\end{array}$ & $\begin{array}{l}\text { Cane yield } \\
\text { (ton/ha) }\end{array}$ & $\begin{array}{l}\text { Sucrose } \\
\text { percent } \\
\text { cane (\%) }\end{array}$ & $\begin{array}{l}\text { Sugar yield } \\
\text { (ton/ha) }\end{array}$ & Pol (\%) & Brix & Purity \\
\hline Conventional & $2.47^{a}$ & $1.46^{\mathrm{a}}$ & $2.57^{a}$ & $150193 . I^{\mathrm{a}}$ & $1219.28 \mathrm{I}^{\mathrm{a}}$ & $10.96^{a}$ & $238.69^{a}$ & $15.80^{\mathrm{a}}$ & $17.70^{\mathrm{a}}$ & $88.99^{a}$ \\
\hline
\end{tabular}

Table 3 Comparison of sugarcane genotypes NCo-334, B52-298 and NI4

\begin{tabular}{|c|c|c|c|c|c|c|c|c|c|c|}
\hline Genotypes & $\begin{array}{l}\text { Stalk } \\
\text { height } \\
\text { (m) }\end{array}$ & $\begin{array}{l}\text { Single } \\
\text { cane } \\
\text { weight } \\
\text { (kg) }\end{array}$ & $\begin{array}{l}\text { Diameter } \\
(\mathrm{cm})\end{array}$ & $\begin{array}{l}\text { Stock } \\
\text { population } \\
\text { (Number/ha) }\end{array}$ & $\begin{array}{l}\text { Cane yield } \\
\text { (ton/ha) }\end{array}$ & $\begin{array}{l}\text { Sucrose } \\
\text { percent } \\
\text { cane (\%) }\end{array}$ & $\begin{array}{l}\text { Sugar yield } \\
\text { (ton/ha) }\end{array}$ & Pol (\%) & Brix & Purity \\
\hline NCo-334 & $2.54^{a}$ & $1.30^{\mathrm{a}}$ & $2.42^{c}$ & $|524| 3.79^{b}$ & $198.137 .93^{b}$ & $10.22^{c}$ & $204.52^{\mathrm{b}}$ & $\mid 4.8 I^{c}$ & $16.68^{c}$ & $88.36^{b}$ \\
\hline B52-298 & $2.20^{c}$ & $1.34^{\mathrm{a}}$ & $2.64^{\mathrm{a}}$ & $1324 \mid 3.79^{c}$ & I $77.434 .49^{\circ}$ & $11.68^{\mathrm{a}}$ & $200.55^{c}$ & $16.42^{\mathrm{a}}$ & $18.48^{a}$ & $88.62^{b}$ \\
\hline NI4 & $2.43^{b}$ & $1.39^{a}$ & $2.57^{b}$ & $160920.68^{a}$ & $223.679 .75^{a}$ & $11.27^{\mathrm{b}}$ & $249.6 \mathrm{I}^{\mathrm{a}}$ & $16.13^{b}$ & $\left.17.9\right|^{\mathrm{b}}$ & $89.91^{a}$ \\
\hline
\end{tabular}

Table 4 Separation of significant means for the interaction effects of genotype, seed source and soil type

\begin{tabular}{|c|c|c|c|c|c|c|c|}
\hline \multicolumn{8}{|l|}{ Mean士SE } \\
\hline Genotype & Seed source & Soil type & $\begin{array}{l}\text { Stalk diameter } \\
(\mathrm{cm})\end{array}$ & $\begin{array}{l}\text { Cane yield } \\
\text { (ton/ha) }\end{array}$ & $\begin{array}{l}\text { Sucrose percent } \\
\text { cane }(\%)\end{array}$ & Pol (\%) & Brix \\
\hline \multirow{4}{*}{ B52-298 } & \multirow{2}{*}{ Tissue culture } & Luvisol & $2.72 \pm 0.09^{b}$ & $105.876 \pm 2.83^{k}$ & $13.23 \pm 0.63^{\mathrm{a}}$ & $18.62 \pm 0.76^{\mathrm{a}}$ & $20.23 \pm 0.60^{\mathrm{a}}$ \\
\hline & & Vertisol & $2.52 \pm 0.09^{d}$ & $176.552 \pm 2.83^{i}$ & $9.75 \pm 0.63^{i}$ & $14.52 \pm 0.76^{g}$ & $\mid 7.01 \pm 0.60^{\mathrm{h}}$ \\
\hline & \multirow{2}{*}{ Conventional } & Luvisol & $2.7 \mathrm{I} \pm 0.09^{b}$ & $221.279 \pm 2.83^{d}$ & $10.87 \pm 0.63^{f}$ & $15.72 \pm 0.76^{d}$ & $17.73 \pm 0.60^{f}$ \\
\hline & & Vertisol & $2.60 \pm 0.09 c$ & $220.114 \pm 2.83^{\mathrm{de}}$ & $11.62 \pm 0.63^{c}$ & $|6.8| \pm 0.76^{\mathrm{ab}}$ & $18.97 \pm 0.60^{b}$ \\
\hline
\end{tabular}


Table Continued.

Mean \pm SE

\begin{tabular}{|c|c|c|c|c|c|c|c|}
\hline Genotype & Seed source & Soil type & $\begin{array}{l}\text { Stalk diameter } \\
(\mathrm{cm})\end{array}$ & $\begin{array}{l}\text { Cane yield } \\
\text { (ton/ha) }\end{array}$ & $\begin{array}{l}\text { Sucrose percent } \\
\text { cane }(\%)\end{array}$ & Pol (\%) & Brix \\
\hline \multirow{4}{*}{ NI4 } & \multirow{2}{*}{ Tissue culture } & Luvisol & $2.53 \pm 0.09^{d}$ & $233.028 \pm 2.83^{c}$ & $10.86 \pm 0.63^{f}$ & $15.50 \pm 0.76^{\mathrm{e}}$ & $17.07 \pm 0.60^{h}$ \\
\hline & & Vertisol & $2.36 \pm 0.09^{f}$ & $217.355 \pm 2.83^{\mathrm{e}}$ & $11.01 \pm 0.63^{\mathrm{e}}$ & $16.09 \pm 0.76^{c}$ & $18.42 \pm 0.60^{c}$ \\
\hline & \multirow{2}{*}{ Conventional } & Luvisol & $2.6 I \pm 0.09^{c}$ & $184.893 \pm 2.83^{h}$ & $11.95 \pm 0.63^{\mathrm{b}}$ & $16.69 \pm 0.76^{b}$ & $17.92 \pm 0.60^{\mathrm{e}}$ \\
\hline & & Vertisol & $2.79 \pm 0.09^{\mathrm{a}}$ & $255.286 \pm 2.83^{a}$ & $11.26 \pm 0.63^{d}$ & $16.24 \pm 0.76^{c}$ & $18.24 \pm 0.60^{d}$ \\
\hline \multirow{4}{*}{ NCo-334 } & \multirow{2}{*}{ Tissue culture } & Luvisol & $2.47 \pm 0.09^{e}$ & $|70.23| \pm 2.83^{i}$ & $10.59 \pm 0.63^{g}$ & $14.99 \pm 0.76^{f}$ & $16.42 \pm 0.60^{i}$ \\
\hline & & Vertisol & $2.45 \pm 0.09^{e}$ & $196.666 \pm 2.83^{f}$ & $10.25 \pm 0.63^{i}$ & $14.92 \pm 0.76^{f}$ & $16.99 \pm 0.60^{i}$ \\
\hline & \multirow{2}{*}{ Conventional } & Luvisol & $2.50 \pm 0.09^{d}$ & $189.117 \pm 2.83^{g}$ & $9.57 \pm 0.63^{k}$ & $13.97 \pm 0.76^{h}$ & $15.96 \pm 0.60^{k}$ \\
\hline & & Vertisol & $2.25 \pm 0.09 \mathrm{~g}$ & $242.069 \pm 2.83^{b}$ & $10.49 \pm 0.63^{\mathrm{h}}$ & $15.35 \pm 0.76^{e}$ & $17.35 \pm 0.60^{g}$ \\
\hline
\end{tabular}

In sugarcane genotype N14, conventional seed sources gave better stock diameter $(\mathrm{cm})(2.61 \pm 0.09)$; sucrose percent cane $(\%)$ (11.95 \pm 0.63$)$; Pol (\%) (16.69 \pm 0.76$)$ and Brix $(17.92 \pm 0.60)$ than tissue culture seed source on Luvisol. However; better cane yield was obtained from tissue culture seed source for N14 on Luvisol. On Vertisol, except for the response variables pol (\%) and Brix, N14 from conventional seed source gave more stock diameter $(\mathrm{cm})$ (2.79 \pm 0.09$)$; cane yield (ton/ha) $(255.286 \pm 2.83)$; and sucrose percent cane (\%) (11.26 \pm 0.63$)$ than tissue culture seed source (Table 4). On Luvisol, sugarcane genotype NCo-334 from tissue culture seed source gave better sucrose percent cane (\%) (10.59 \pm 0.63$)$; Pol (\%) $(14.99 \pm 0.76)$ and Brix $(16.42 \pm 0.60)$ while it is less in stalk diameter (cm) $(2.47 \pm 0.09)$ and cane yield (ton/ha) $(170.231 \pm 2.83)$ than the conventional seed source. On Vertisol, except in stalk diameter, NCo334 from conventional seed source showed better result in cane yield, sucrose percent cane, Pol\% and Brix (Table 4).

Generally, comparison of the two seed sources as initial seed cane, in sugarcane genotypes B52-298 and NCo-334, on both soil types, the cane yield (ton/ha) obtained from tissue culture seed source is inferior to the conventional seed source and hence the rate of propagation. However, better cane yield (ton/ha) from tissue culture seed source was obtained in N14 on Luvisol; 233.028 ton/ha from tissue culture seed source against 184.893 ton/ha from conventional seed source; 48.135 tons more cane yield per ha (Table 4). To plant one hectare of land with tissue culture plantlets, it requires about
$52,500 \mathrm{Br}$. (2625US\$) (40 cm spacing, $3 \mathrm{Br} /$ plantlet) while it requires about $6750 \mathrm{Br}$ (137.5US\$) to cover with sett from conventional seed source (seed rate: 120-150 Quintal/ha; $50 \mathrm{Br} / \mathrm{Quintal}$ ). The estimated equivalent value of 48.135 tons of seed cane is about $24,067.50 \mathrm{Br}$ (1203.4US\$). Even if tissue culture seed sources of N14 on Luvisol seems to give better cane yield, comparison of the cost of the two seed sources indicates tissue culture seed source incurred an extra cost of Br. 21,682.50 (US\$1084.13) per hectare. Thus, use of tissue culture seed sources of B52-198, NCo-334 and N14 as initial sources of seed cane under the then secondary acclimatization procedure, similar agronomic management practices and quality control at Matahara Sugarcane plantation is not viable. For the response variables Stalk height (cm), Stalk population (Number/ha), Purity (\%) and Sugar yield (ton/ha), the interaction effects of genotype and seed source is highly significant. In sugarcane genotype N14, sugarcane plants from tissue culture showed better stalk height $(\mathrm{cm})$ and stock population (Number/ ha) than their donors from conventional seed source while the results obtained from tissue culture seed sources in genotypes $\mathrm{NCo}-334$ and B52-198 was inferior to the results from the conventional source for the two response variables(stock height and population) (Table 5). Nevertheless, estimated sugar yield (ton/ha) from tissue culture seed source sugarcane plants is less than the conventional seed source plants for all the three genotypes. Similarly, except in genotype $\mathrm{NCo}-$ 334 , the percent purity of tissue culture plants is not better than that of the conventional seed source plants in any of the genotypes tested (Table 5).

Table 5 Separation of means for the interaction effects of genotype by seed source

\begin{tabular}{|c|c|c|c|c|c|}
\hline \multirow{2}{*}{ Genotype } & \multirow{2}{*}{ Seed source } & \multicolumn{4}{|l|}{ Mean \pm SE } \\
\hline & & Stalk height $(\mathrm{cm})$ & Stalk population (Number/ha) & Sugar yield (ton/ha) & Purity (\%) \\
\hline \multirow{2}{*}{ B52-298 } & Tissue culture & $1.93 \pm 0.12^{\mathrm{e}}$ & $119138 \pm 2.53^{f}$ & $155.83 \pm 0.44^{e}$ & $88.68 \pm 0.90$ \\
\hline & Conventional & $2.47 \pm 0.12^{c}$ & $145690 \pm 2.53^{c}$ & $245.26 \pm 0.44^{b}$ & $88.56 \pm 0.90^{\circ}$ \\
\hline \multirow{3}{*}{ NI4 } & Tissue culture & $2.50 \pm 0.12^{\mathrm{ab}}$ & $|7620| \pm 2.53^{\mathrm{a}}$ & $245.28 \pm 0.44^{b}$ & $88.78 \pm 0.90$ \\
\hline & & & & & \\
\hline & Conventional & $2.36 \pm 0.12^{\mathrm{d}}$ & $|4562| \pm 2.53^{d}$ & $253.95 \pm 0.44^{a}$ & $91.04 \pm 0.90^{\mathrm{a}}$ \\
\hline \multirow{3}{*}{ NCo-334 } & Tissue culture & $2.49 \pm 0.12^{b}$ & $145586 \pm 2.53^{\mathrm{e}}$ & $192.19 \pm 0.44^{\mathrm{d}}$ & $89.53 \pm 0.90^{b}$ \\
\hline & & & & & \\
\hline & Conventional & $2.59 \pm 0.12^{\mathrm{a}}$ & $|5924| \pm 0.53^{b}$ & $216.86 \pm 0.44^{c}$ & $87.19 \pm 0.90^{\circ}$ \\
\hline
\end{tabular}




\section{Conclusion}

Cane yield (ton/ha) obtained from tissue culture seed sources of B52-298 and NCo-334 was inferior to the conventional seed sources and hence the rate of propagation. In N14, tissue culture seed sources produced 48.135 tons more cane per hectare than the conventional seed source. However, even considering the better cane yield obtained in N14, the cost of tissue culture plantlets is by far costly than that of the conventional seed source. Thus, Comparison of tissue culture and conventional seed sources as initial source of seed cane for all the three sugarcane genotypes: B52-298, NCo-334 and N14 tested on Luvisol and Vertisol types under the then acclimatization and field agronomic management practices of Matahara Sugar Estate revealed that tissue culture seed sources have no direct benefit than the conventional seed sources. The reduced or equivalent performance of tissue culture seed sources could probably be due to the non standard secondary acclimatization; difficulty in field establishment of the bare rooted plantlets and lack of facility to test the genetic fidelity and disease freeness of the tissue culture seed sources at different micro propagation stages. In addition, owing to their delicate nature, tissue culture plantlets may require their own separate agronomic management practices.

\section{Acknowledgements}

None.

\section{Conflict of interest}

The author declares no conflict of interest.

\section{References}

1. Sharma M. In vitro regeneration studies of sugarcane. Patiala, India; 2005. p. 24-32.
2. Daniels J, Roach BT. Taxonomy and Evolution. In: DJ Heinz editor. Sugarcane improvement through breeding; 1987.

3. Asano T, Takahashi S, Tsudzuki T, et al. Complete nucleotide sequence of the sugarcane (Saccharum officinarum) chloroplast genome: A comparative analysis of four monocot chloroplast genomes. DNA Res. 2004;11(2):93-99.

4. Singh N, Kumar A, Garg GK. Genotype influence of phytohormone combination and sub culturing on Micropropagation of sugarcane varieties. Indian Journal of biotechnology. 2006;5:99-106.

5. Food and Agriculture Organization of the United States of America. World sugarcane production statistics. FAO; 2013.

6. Sengar K, Sengar RS, Garj SK. Developing an efficient protocol through tissue culture technique for sugarcane Micropropagation; 2010.

7. Ramanand, Lal M, Singh SB. Comparative performance of micro propagated and conventionally raised crops of sugarcane. Sugar tech. 2005;7(2):93-95.

8. Comstock JC, Miller JD. Yield comparison: Disease free tissue cultures versus bud propagated planted sugarcane plants and healthy versus yellow leaf virus infected plants. Journal American Society Sugar Cane Technologists. 2004;24:1-10.

9. Bailey RA, Bechet GR. a comparison of seed cane derived from tissue culture with conventional seed cane. Proceedings of the South African Sugar Technologists' Association. 1989. p. 1-5.

10. Sood N, Gupta PK, Srivastava RK, et al. Comparative studies on field performance of micro propagated and conventionally propagated sugarcane plants. Plant Tissue Culture and Biotechnology. 2006;16(1):25-29.

11. Ibrahim M, Tolera B, Aman J, et al. Evaluation of tissue culture raised sugarcane planting materials against their donor conventional seed sources as initial source of seed cane at tendaho sugar development project, North-Eastern Ethiopia. J Horticulture. 2016;3:168. 